\title{
Critical appraisal of cardiac implantable electronic devices: complications and management
}

\author{
This article was published in the following Dove Press journal: \\ Medical Devices: Evidence and Research \\ 8 September 201 I \\ Number of times this article has been viewed
}

\author{
Luigi Padeletti' \\ Giosuè Mascioli² \\ Alessandro Paoletti Perini' \\ Gino Grifoni' \\ Laura Perrotta' \\ Procolo Marchese ${ }^{3}$ \\ Luca Bontempi ${ }^{3}$ \\ Antonio Curnis ${ }^{3}$ \\ 'Istituto di Clinica Medica e \\ Cardiologia, Università degli Studi \\ di Firenze, Italia; ${ }^{2}$ Elettrofisiologia, \\ Istituto Humanitas Gavazzeni, \\ Bergamo, Italia; ${ }^{3}$ Elettrofisiologia, \\ Spedali Civili, Brescia, Italia
}

Correspondence: Luigi Padeletti Istituto di Clinica Medica e Cardiologia, Università degli Studi di Firenze.

Viale Morgagni 85, 50 I 34, Firenze, Italia

$\mathrm{Tel}+390557947514$

Fax +390557947850

Email lpadeletti@interfree.it
Abstract: Population aging and broader indications for the implant of cardiac implantable electronic devices (CIEDs) are the main reasons for the continuous increase in the use of pacemakers (PMs), implantable cardioverter-defibrillators (ICDs) and devices for cardiac resynchronization therapy (CRT-P, CRT-D). The growing burden of comorbidities in CIED patients, the greater complexity of the devices, and the increased duration of procedures have led to an augmented risk of infections, which is out of proportion to the increase in implantation rate. CIED infections are an ominous condition, which often implies the necessity of hospitalization and carries an augmented risk of in-hospital death. Their clinical presentation may be either at pocket or at endocardial level, but they can also manifest themselves with lone bacteremia. The management of these infections requires the complete removal of the device and subsequent, specific, antibiotic therapy. CIED failures are monitored by competent public authorities, that require physicians to alert them to any failures, and that suggest the opportune strategies for their management. Although the replacement of all potentially affected devices is often suggested, common practice indicates the replacement of only a minority of devices, as close follow-up of the patients involved may be a safer strategy. Implantation of a PM or an ICD may cause problems in the patients' psychosocial adaptation and quality of life, and may contribute to the development of affective disorders. Clinicians are usually unaware of the psychosocial impact of implanted PMs and ICDs. The main difference between PM and ICD patients is the latter's dramatic experience of receiving a shock. Technological improvements and new clinical evidences may help reduce the total burden of shocks. A specific supporting team, providing psychosocial help, may contribute to improving patient quality of life.

Keywords: pacemaker, cardiac cardioverter-defibrillator, cardiac implantable electronic devices, infection, recall, quality of life

\section{The emerging use of cardiac implantable electronic devices}

At the beginning of the 1950s, Zoll described the possibility of preventing death or syncope in Stokes-Adams syndrome by applying an external cardiac device, able to pace the heart. ${ }^{1}$ Six years later, Elmqvist and Senning implanted the first cardiac pacemaker (PM) by thoracotomy. ${ }^{2}$ Since then, the use of PMs in the management of cardiac diseases has constantly increased, while their software and hardware have been continuously developing. The first implantable cardioverter-defibrillator (ICD) was implanted in $1980 .{ }^{3}$

Population aging and broader indications for the implant of cardiac implantable electronic devices (CIEDs), due to the publication of randomized multicenter 
trials, such as the MADIT II, the SCD-HeFT, the COMPANION and the CARE-HF, ${ }^{4-7}$ are the main reasons for the continuous increase in the use of PMs, ICDs, and devices for cardiac resynchronization therapy (CRT-P, CRT-D). The latest figures derived from the Eucomed Data show that since 2005 to 2008 the rate of implant has continued to increase in the countries of the European Community, with a higher increase for ICDs than for PMs. ${ }^{8}$ The implant rate for one million inhabitants increased between 2005 and 2008 for PMs from 728.8 to 810.8 , for ICDs from 79.6 to 119.4, for CRT-P devices from 24.3 to 23.6, and for CRT-D devices from 28.1 to 53.4. In the United States, between 1997 and 2004, implantation rates increased by 19\% for PMs and by $60 \%$ for ICDs, with about $70 \%$ of implanted patients being 70 years or older. ${ }^{9}$ In the next 2 years, a further increase of $12 \%$ in overall CIED implantations (PMs and ICDs) was documented, with 222,940 new implanted devices in 2006, according to data from the National Hospital Discharge Survey. ${ }^{10}$

In the coming years, an increase in patient life-length will mean that dealing with patients with CIEDs will be more and more common for every physician, including "noncardiologist" physicians.

\section{Overview of the design and basic working mechanism of CIEDs}

The most commonly used CIEDs are PMs and ICDs. CRT-P and CRT-D devices should be regarded as special PMs (CRT-P) or ICDs (CRT-D), and therefore they do not deserve a specific description.

A PM consists mainly of a lithium iodine battery, an integrated circuit, and 1 to 3 electrodes that connect the generator to the heart, whose function is "to sense" the cardiac intrinsic electrical activity and "to pace" the cardiac chambers, when necessary ${ }^{11}$ (Figure 1). The lithium iodine battery is currently preferred to different battery chemistries, because it provides a steady voltage during its whole life and has a predictable decay when approaching its end of life, so allowing the physician to choose the most favorable moment for the elective replacement of the device. ${ }^{12}$ Moreover, most modern PMs are equipped with special algorithms which can automatically detect the lowest energy necessary to pace the heart, in order to minimize the pacing outputs and prolong the battery life.

Depending on clinical needs, one electrode is used, either in the atrium or in the ventricle (SSI mode), or two electrodes, in which case the device will pace and sense both the atrium and the ventricle (DDD mode). Three electrodes are used

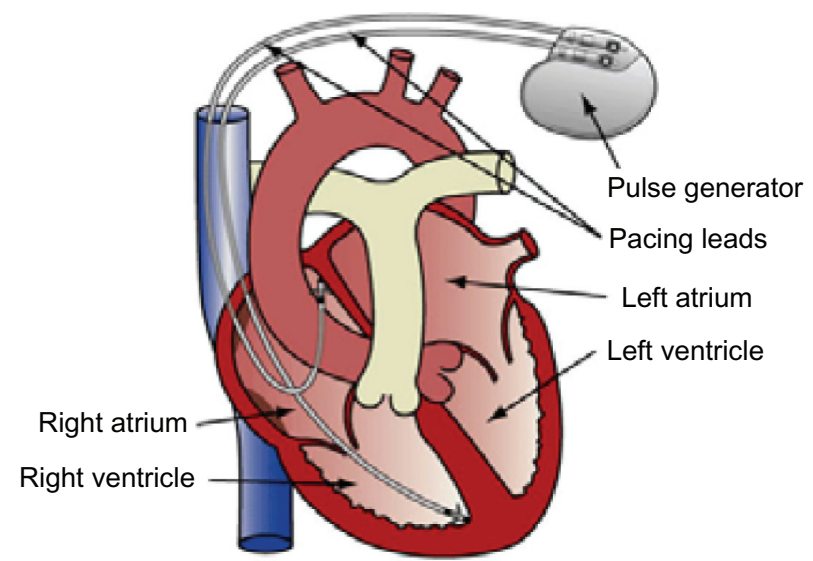

Figure I An example of bicameral pacemaker. Leads are inserted into the right cardiac sections via the superior vena cava.

when pacing therapy involves the right atrium and both the right and the left ventricle: this modality is called "biventricular pacing," and is reserved for patients affected by dilated cardiomyopathy and severe impairment of the left ventricular systolic function. ${ }^{6,7}$ To date, all PMs work "on demand": in other words, they do not pace the heart if the spontaneous heart rate does not fall below a critical level, which can be programmed by the physician according to the patient's needs. In order to achieve this result, one of the most important components of the integrated circuit within the device is a special counter, which measures the interval in milliseconds between the native beats: if this interval exceeds the one programmed as "lower rate" the device will deliver an electrical pulse that can make the heart beat. PMs can be equipped also with special built-in electronic sensors able to detect the patient's motion and/or breathing and to define, by specific algorithms and settings, a new pacing rate automatically adjusted according to their metabolic demand. This sensor will be used for patients with chronotropic incompetence. Besides, many PMs have special algorithms to minimize the right ventricle pacing, which has been proved to cause ventricular desynchronization, reduced pump function, and a higher burden of atrial fibrillation. ${ }^{13-16}$ The two main strategies to allow the spontaneous ventricular contraction are the extension of the atrial-ventricular interval in DDD mode (Figure 2), and the preference for single-chamber atrial pacing (AAI mode) with an automatic switch to dual-chamber pacing (DDD mode), should the spontaneous ventricular beat be missing. ${ }^{17}$

ICDs are more sophisticated devices. While their functioning in case of bradyarrhythmia is exactly the same as for PMs, their main feature is the capability to interrupt ventricular tachyarrhythmias, such as ventricular tachycardia (VT) and ventricular fibrillation (VF), restoring 


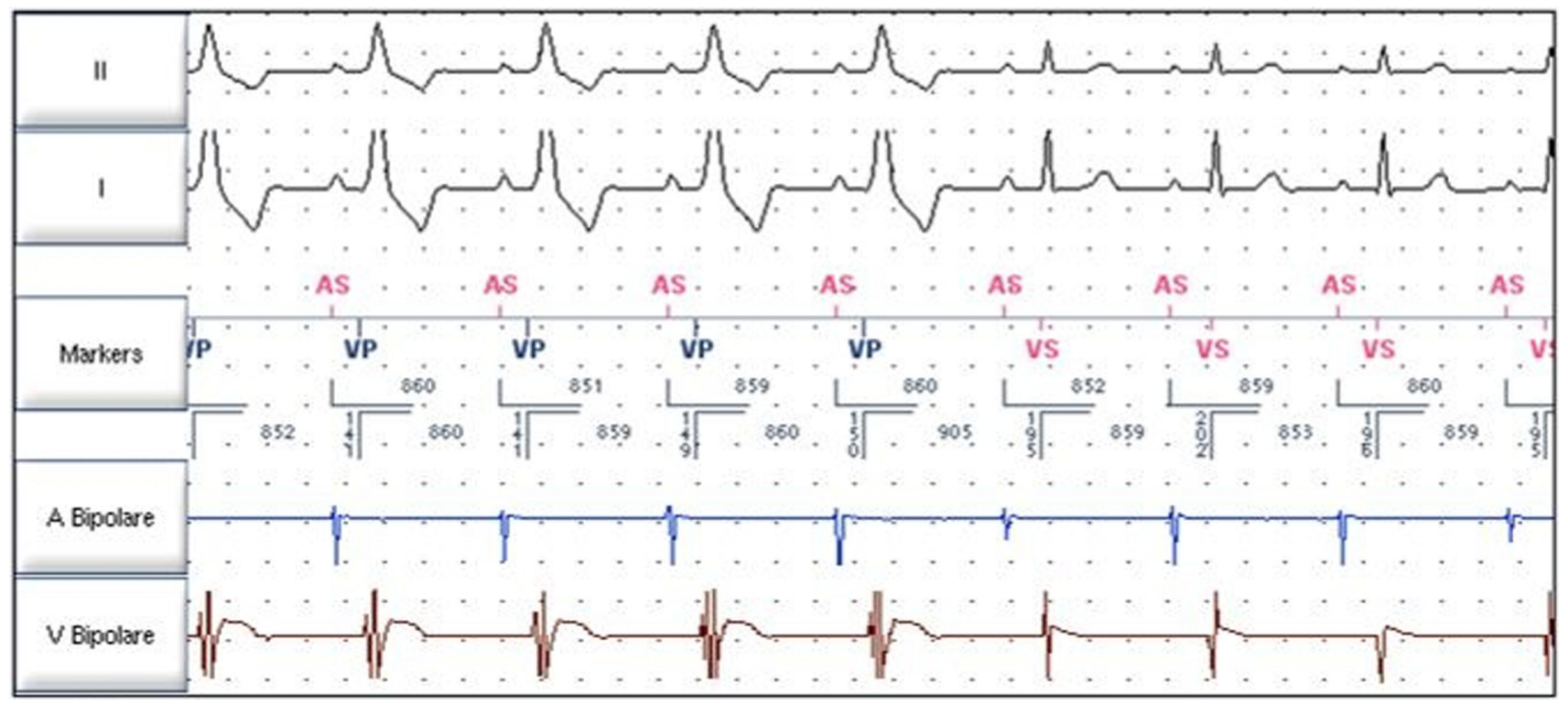

Figure 2 Extension of the A-V delay to permit the rise of the spontaneous atrial-ventricular conduction. In the upper part, standard ECG leads DI and DII are shown. In the middle, markers indicate the spontaneous atrial (AS), the paced ventricular (VP) and the spontaneous ventricular (VS) cardiac activity. Intervals between consecutive complexes are also expressed. In the lower part, atrial and ventricular electrocardiograms are shown. When the A-V delay is prolonged from I50 to I 95 milliseconds, the spontaneous ventricular activity appears. From the standard ECG leads it can be seen that the ventricular sensed complexes are fully spontaneous and not fusion beats. Note: Reproduced with permission of St Jude Medical Inc, St Paul, Minnesota 55I I7, USA.

the sinus rhythm. ${ }^{18}$ ICDs are nowadays used for secondary prevention in patients resuscitated from sudden cardiac death and for primary prevention in patients at high risk for malignant ventricular arrhythmias (VAs). ${ }^{4-6,19,20}$ As for PMs, one of the most important components of an ICD is the battery, which requires a specific chemistry designed to last a long time and to deliver a high amount of current in a short time, when the device has to deliver shock therapies. The integrated circuit related to pacing and sensing functions is similar to that of a PM. ICDs have additional circuitry, called a high voltage circuit, which can charge the high energy capacitor used to treat tachyarrhythmias. If a high ventricular heart rate is detected, the internal counter starts counting the rate and the number of beats, until the predefined criteria (both heart rate and event duration, in seconds or in number of beats) for a ventricular arrhythmia are satisfied. The device can also perform a deeper analysis of the arrhythmia, in order to discriminate between supraventricular tachyarrhythmias and ventricular ones. This further analysis is conducted on the basis of parameters such as the presence of atrial-ventricular dissociation, the onset of the arrhythmia, which can be sudden or progressive, the stability of $\mathrm{R}-\mathrm{R}$ intervals, and, in the most recent devices, the morphologic discrepancies between the native impulse and the one detected during the arrhythmic event. To interrupt the arrhythmia, the ICD can fire a burst of pulses at a frequency that is faster than the spontaneous heart rate, in order to "capture" the tachycardia and to stop it (the socalled "anti-tachycardia pacing" ATP). This treatment is safe and effective in interrupting the arrhythmia in a very high percentage $(>90 \%)$ of $\mathrm{VTs}^{21}$ and in more than $70 \%$ of fast VTs (ie, 188 up to $250 \mathrm{bpm}$ ). ${ }^{22}$ In case the attempt is ineffective, or when particularly fast VAs or VFs occur, the ICD can "fire" a shock: within the high energy capacitor, an energy of about 30-40 Joules is stored and then fired to the right ventricle in a biphasic wave between the electrode and the can of the device itself or even in a reversed mode ("can to coil") (Figure 3). Modern devices can be programmed to define the energy level and the sequence of shock attempts. To treat a single episode of VA, 6 to 8 shocks are available, according to different ICD models. If the arrhythmia persists, even after the last shock delivery, an external intervention is required to restore the sinus rhythm. ICDs can store in their memory, for later review, several arrhythmic episodes with their related electrograms (like Holter recordings).

Both ICDs and PMs are equipped with automatic algorithms able to run measurements of electrical parameters of the electrodes and to collect their trends, storing these values for later evaluation. Moreover, modern devices have the capability to send to the physician, through a remote connection (web, fax, telephone), stored data or warning messages related to the system diagnostic and clinical events. 


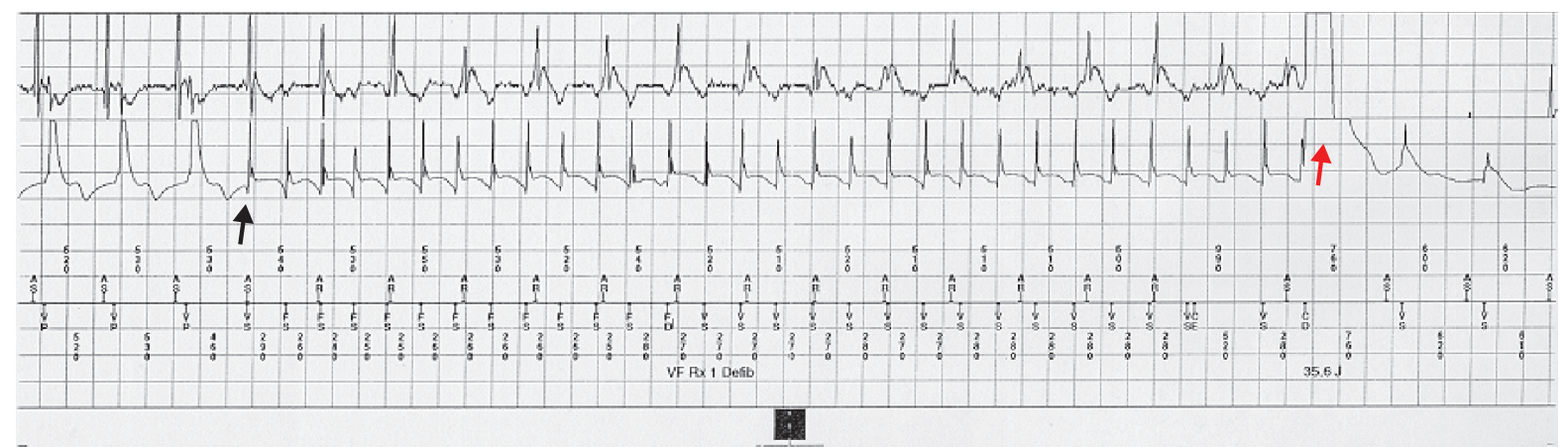

Figure 3 Example of a fast ventricular tachycardia $(>210 \mathrm{bpm})$ detected by the implantable cardioverter-defibrillator (ICD) in the ventricular fibrillation zone, treated efficaciously with a DC-shock. In the superior part, the registration of intracardiac electrograms is shown: atrial (upper trace) and ventricular (lower trace) activities are dissociated. In the inferior part, the arrhythmia is expressed as intervals between each detected complex: fast ventricular rate and atrial-ventricular dissociation are detectable. The black arrow shows the onset of the ventricular arrhythmia; the red arrow indicates the shock provided by the device, which restores the sinus rhythm. Reproduced with permission of Medtronic Inc, Minneapolis, Minnesota 55432, USA.

\section{Patient's history and prevalence of infections in use of CEIDs}

Infections in a patient implanted with a CIED may involve either the pocket or the electrodes and the endocardium. ${ }^{23}$ Their clinical presentation at pocket level includes signs such as erythema, warmth, fluctuation, wound dehiscence, tenderness, purulent drainage, or erosion of generator or leads through the skin (Figure 4), while the presence of CIED-related endocarditis is confirmed when valvular or lead vegetations are detected by echocardiography, or if the Duke criteria for infective endocarditis are met $^{24}$ (Figure 5). At least 2 sets of blood cultures should be obtained before the initiation of antimicrobial therapy in all patients in whom a CIED infection is suspected. ${ }^{25}$ As the presentation can be indolent, a high degree of clinical suspicion is required. This is of paramount importance in those CIED patients presenting with Staphylococcus aureus bacteremia (SAB), as positive blood cultures may be the sole sign of device

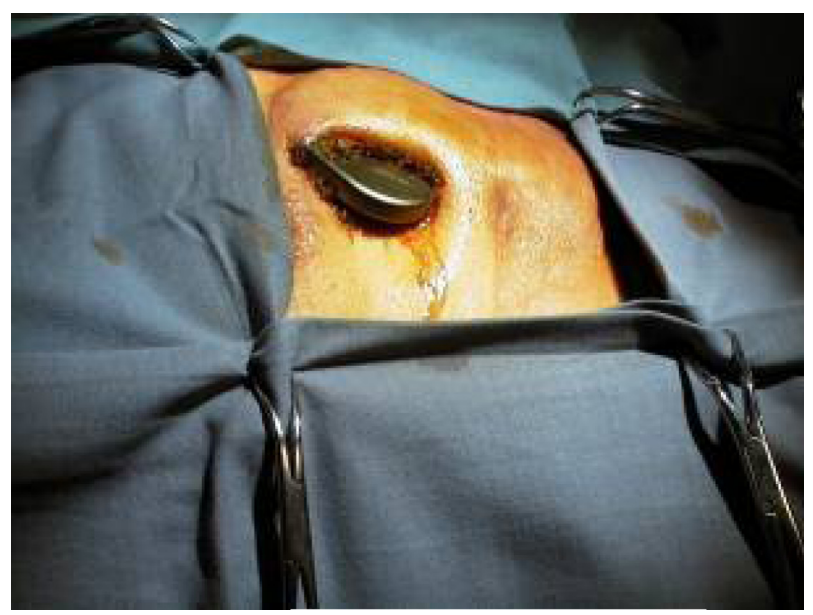

Figure 4 An example of cardiac implantable electronic device infection at pocket level. infection. ${ }^{26}$ In recent years, the number of implanted CIEDs has increased constantly, and CIED infections have also been increasing at higher rates: in the United States, from 1996 to 2003, infections increased 3.1-fold, but total implantations only 0.49 -fold; ${ }^{10}$ between 2004 and 2006, CIED infections increased by $57 \%$, whereas implantations increased by only $12 \% .{ }^{27}$ ICDs have been reported to be more liable to cause infections than PMs, ${ }^{28}$ and hospitalizations due to ICD infections are increasing at a higher rate. ${ }^{27}$ The presence of device infections in hospitalized CIED patients is an ominous condition, as these patients have longer lengths of stay and up to five-fold risk of in-hospital death $(P<0.001$ for both),${ }^{10}$ with high rates of one-year mortality $(17 \%)$, even after the removal of the device. ${ }^{29}$

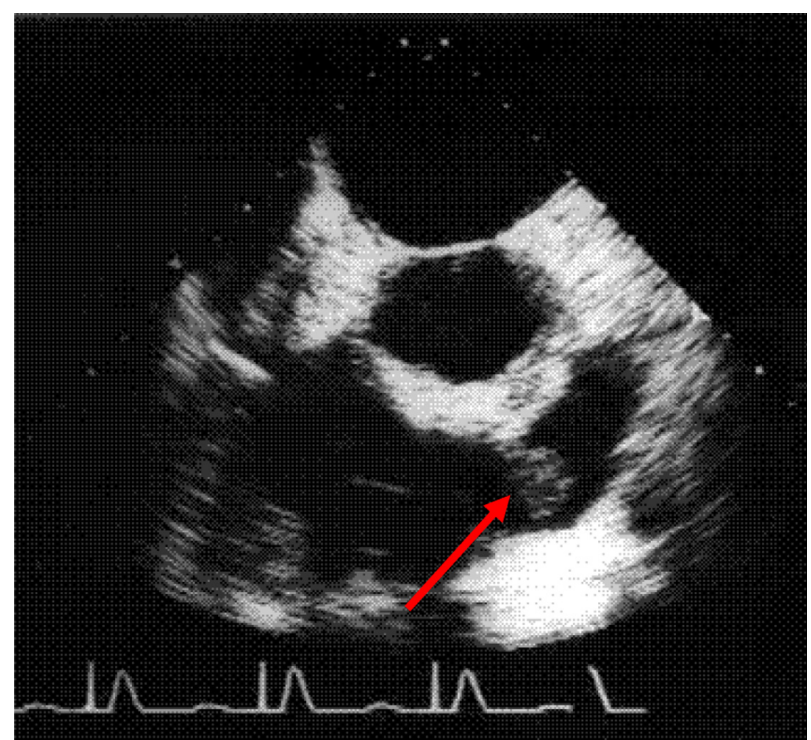

Figure 5 Endocarditis on native pulmonary valve, as detected at transesophageal echocardiography. The arrow indicates the presence of vegetations on the pulmonary valve. 
The development of an infection is the result of the interaction among the patient, the device, and the infective microorganisms, mostly bacteria. The patient-related risk factors include the presence of comorbidities which lead to immunosuppression, such as the use of corticosteroids, ${ }^{23}$ or the presence of renal dysfunction ${ }^{30}$ up to end-stage renal disease, ${ }^{31}$ the need of anticoagulant therapy,$^{30}$ and the presence of fever within the previous 24 hours, ${ }^{32}$ whereas the use of antibiotic prophylaxis within the previous 2 hours (mostly cefazoline) has been proven to prevent the occurrence of infections..$^{23,32,33}$ According to the analysis by Voigt et al on the data from the National Hospital Discharge Survey database, ${ }^{10}$ from 1996 to 2006, hospitalization for CIED implantation has become slightly, but significantly, shorter, while the patient burden of severe comorbidities, such as diabetes mellitus and organ failure, has significantly increased. Among the peri-procedural factors which can favor an infection, the most common, apart from not using antibiotic prophylaxis, are the number of implanted leads, ${ }^{23,34}$ the procedure type, ${ }^{31}$ the operator's experience, ${ }^{35}$ the performance of a device revision or replacement versus a de novo implant and, above all, the need for early re-intervention. ${ }^{32,34}$ This last situation carries the greatest risk, ranging across different studies, from $3.67-{ }^{30}$ to 15-fold ${ }^{32}$ (Table 1).

The kind of microorganism involved plays a major role in determining the likelihood of CIED infections: in fact, as already mentioned, bacteremia can be the sole manifestation of infection, especially for patients presenting with SAB. ${ }^{26}$ In such patients, the likelihood is increased when the device is an ICD; moreover, patients with SAB and a prosthetic cardiac valve have an extremely high risk (up to $50 \%$ ) of a double infection, both of the device and of the leads. ${ }^{26}$ Staphylococci account for $60 \%$ to $80 \%$ of cases in most series, ${ }^{23,29,30}$ and are more likely to cause infections early after the implant, ${ }^{30}$ while later infections are more likely to be determined by other Gram-positive bacteria, by Gram-negative bacteria, ${ }^{36}$ or even to be associated with negative cultures ${ }^{30}$ (Table 2).
Table I Risk factors for CIEDs infections

- Immunosuppression (diabetes, CRF, steroid therapy

and/or immunosuppressive therapy, malignancy)

- No periprocedural antimicrobial prophylaxis

- Fever less than 24 hours before implantation

- Vitamin $\mathrm{K}$ - antagonist therapy

- Postoperative hematoma

- Generator replacement

- Preprocedural temporary pacing

- Need for acute repositioning of a lead

- Presence of abandoned leads

- Operator experience

- Duration of procedure

- Number of implanted leads

Abbreviations: CIEDs, cardiac implantable electronic devices; CRF, chronic renal failure.

As recently stated by the American Heart Association (AHA), ${ }^{25}$ once the diagnosis of CIED infection has been made, removal of both the device and the lead is recommended, because of the high risk of relapse due to retained hardware, ${ }^{25,37}$ even in case of demonstrated valvular endocarditis without definite involvement of the leads or the device, and in case of persisting or relapsing SAB. ${ }^{25}$ Antibiotic treatment is mandatory after removal has been performed. In case of persistent Gram-negative bacteraemia despite appropriate antibiotic therapy, CIED removal is a reasonable option. However, when the infection at pocket site is only superficial or incisional, and there is no involvement of the device, there is no indication to remove it, as a cycle of oral antibiotic therapy against Staphylococci is regarded as reasonably effective. ${ }^{25}$ The removal of the device can be performed surgically or using a percutaneous approach, which can be regarded as the treatment of choice in expert hands at tertiary referral centers, with rates of mortality directly due to the procedure lower than $0,5 \%{ }^{29}$ The timing for re-implantation still varies among different centers, ${ }^{25,29,37}$ but relapses are not uncommon, and are more frequent when the new implant is performed during the

Table 2 Microbiology of PM/ICD infections

\begin{tabular}{|c|c|c|}
\hline $\begin{array}{l}\text { Staphylococcal CIED-related infections } \\
(70 \%-90 \%)\end{array}$ & $\begin{array}{l}\text { Non-staphylococcal CIED-related infections } \\
(10 \%-30 \%)\end{array}$ & \\
\hline - S. aureus (early infections) & - Enterococci & Negative cultures (5\%-20\%) \\
\hline \multirow[t]{8}{*}{ - S. epidermidis (late infections) } & - Streptococci & Polymicrobial (2\%-7\%) \\
\hline & - Proteus & \\
\hline & - Klebsiella & \\
\hline & - E. coli & \\
\hline & - Pseudomonas aeruginosa & \\
\hline & - Salmonella & \\
\hline & - Mycobacteria & \\
\hline & - Mycetes (rare) & \\
\hline
\end{tabular}

Abbreviations: CIED, cardiac implantable electronic device; ICD, implantable cardioverter-defibrillator; PM, pacemaker. 
same hospitalization. ${ }^{29}$ According to AHA guidelines, ${ }^{25}$ this procedure must be performed no sooner than 72 hours after negative blood cultures, but must be delayed by 2 weeks in case of demonstration of infectious involvement of a native valve. As the most critical moment for the development of infections is the implant, ${ }^{38}$ new strategies are being investigated in an effort to reduce bacterial contamination at pocket site. A recent retrospective multicenter study has assessed the feasibility, in a population of patients at high risk for CIED infections, of implanting the device together with a polymer mesh, which releases minocycline and rifampin, in addition to standard systemic antimicrobial prophylaxis (cephalosporin or vancomycin). ${ }^{39}$ This procedure has proved to be feasible, as it did not reduce the rate of successful implantations, and seemed to reduce the likelihood of infections at short-term follow-up (1.9 \pm 2.4 months), when compared with other published studies. ${ }^{32,33,39}$

\section{Efficacy and safety in the use of CEIDS}

The efficacy of ICDs in the reduction of sudden cardiac death has been proven in primary and secondary prevention trials, ${ }^{4,5,19,20}$ while CRT-D and CRT-P devices can reduce both overall and cardiovascular mortality and the number of hospitalizations in heart failure patients; these devices are also able to improve patients' functional capacity and quality of life (QOL). ${ }^{6,7}$ Moreover, it has recently been demonstrated that the advantage of the use of ICDs in preventing sudden cardiac death increases further at long-term follow-up ( 8 years), ${ }^{40}$ while the cost-effectiveness ratio per saved discontinued life-year dramatically improves during the same period. However, CIEDs beget an intrinsic risk of mechanical failure, which can involve either the generator (PM or ICD) or the leads. In recent years, despite enhancements in device technology and manufacturing processes, a sharp increase in manufacturer recalls has been observed. ${ }^{41,42}$ The United States Food and Drug Administration (FDA) classifies medical device recalls into 3 categories, representing the potential risk to public health: Class I, Class II, and Class III, where a Class I recall indicates a high risk of serious adverse health consequences or death and a Class III a low risk. Both the FDA $^{43}$ and the Heart Rhythm Society ${ }^{44}$ have set guidelines for advisories. When an advisory has been issued, patients with an affected device must be contacted and physicians have to choose the best management strategy, considering the estimates of rate of possible malfunction, the likely effect of the issue on specific patients (eg, pacemaker dependency), and the individual center's procedural risk associated with the replacement. Three management strategies are possible: replacing all the recalled devices, replacing a few devices, not replacing any device. According to several analyses, the mean percentage of replaced devices, in different countries, is lower than $20 \%{ }^{41,45}$ while the complications rate ranges from $0.62 \%{ }^{46}$ to $8.1 \%{ }^{41}$ When a device is returned to the manufacturer, laboratory technicians and engineers assess all its functions and perform an analysis through a series of diagnostic tests that verify the performance of defibrillation, pacing, sensing, memory, and recording functions. Test results are compared to original manufacturing records and design intent. Companies should inform the regulatory authorities of each significant event that poses potential risk to patients' health, and periodically publish a performance report, indicating the overall incidence of malfunctions that have occurred for each product.

On 1 July 2005, the FDA issued a class I recall after a notification from Boston Scientific (formerly Guidant) about a potential short circuit that might have affected the Prizm ICD (model 1861), manufactured on or before 16 April 2002 , and about a potential deterioration in the wire insulator within the lead connector block, affecting the Renewal I (model H135) and Renewal II (model H155) CRT-D, manufactured on or before 26 August $2004 .{ }^{47}$ Perrotta et al ${ }^{48}$ have analyzed the experience of 247 Italian centers dealing with this advisory: out of 3185 devices subject to recall, only $18.7 \%$ were explanted $(16.5 \%$ of Prizm and $19.6 \%$ of Renewal devices) and were returned to the company for testing. None of the analyzed Prizm ICDs showed the failure mentioned in the advisory, while 1 out of the $458(0.21 \%)$ tested Renewal devices exhibited it. During the follow-up of patients whose devices had not been replaced, no failures were reported for the Prizm models, whereas 7 out of 2342 $(0.29 \%)$. Renewal devices had been explanted, as they had shown the failure described in the advisory, although the patients involved had not experienced any symptoms. To date, no deaths have been reported by physicians to the Company as potentially associated with the device failure described in the advisory, either for Prizm or for Renewal models. Although only a minority of potentially affected devices has been replaced, this strategy seems to be justified, as the actual failure rate ( $0 \%$ for Prizm, $0.34 \%$ for Renewal) was lower than the estimated one reported in the most recent Company Product Performance Report $(0.72 \%$ for Prizm and $1.83 \%$ for Renewal).

Data also exist from advisories about leads. In 1994, the Teletronics Accufix active fixation leads were subjected to recall because of the risk of fracture and protrusion of the 
$\mathrm{J}$ retention wire, which had caused pericardial tamponade, perforation of the right atrium, embolization to the pulmonary circulation, and a few deaths. Of the potentially affected leads, $13 \%$ were extracted, but the risk of fatal and of life-threatening complications were much higher for extraction than for leaving the lead in place, especially for elderly patients, while the risk of lead fracture and complications were lower in the elderly. ${ }^{49}$

Medtronic Sprint Fidelis implantable cardioverterdefibrillator leads are prone to fracture, which caused inappropriate shocks and a few reported deaths. When compared with Medtronic Sprint Quattro leads, they showed a significantly higher fracture rate $(2.81 \%$ /year vs $0.43 \%$ / year, $P<0.0001) .{ }^{50}$ In a study by Hauser et al $^{50}$ although no deaths or injuries due to lead failure had been reported, up to $42 \%$ of the fractured leads caused inappropriate shocks. Interestingly, the failure rate was higher for younger patients, for women, and for patients affected by channelopathies or by hypertrophic cardiomyopathy: these findings support once more the need for risk stratification in choosing whether to replace a lead or not.

In fact, for both leads and devices, the decision to replace an element subject to recall is still uncertain, as it depends on several variables, among which the most important are the estimated rate of device failure, the arrhythmic risk, and the mortality rate of device replacement. ${ }^{51,52}$ In most cases, a conservative strategy with short (3-month) follow-up may prove to be safer than an attempt to replace the potentially affected element, either because the effective failure rate can be overestimated, ${ }^{48}$ or because of the low benefit/risk ratio for the replacement strategy. ${ }^{49}$

\section{Patient-focused perspectives such as quality of life, patient satisfaction/acceptability}

Implantation of a PM or an ICD in a patient may cause problems in psychosocial adaptation and QOL, and may contribute to the development of affective disorders. PM patients are sometimes able to feel their pacing therapy or may be entirely PM-dependent to maintain a functional heartbeat, but they do not have to contend with the dramatic experience of defibrillation shocks, like many ICD patients; this seems to be the main difference between the two groups in the experience of living with an implanted cardiac device.

Clinicians are usually unaware of the psychosocial impact of implanted PMs and ICDs, as they focus their attention mainly on the technical aspects of device functions rather than on psychosocial factors. For this reason, a better understanding of factors likely to contribute to patients' perception of their own health would be help in their management, and in the training of clinicians and nurses.

One of the first studies to assess differences in psychosocial adaptation, QOL, and incidence of affective disorders between patients with PMs and those with ICDs (shock and no-shock groups) found no difference in scores such as the Hospital Anxiety and Depression (HAD) scale or Short Form 36 QOL measurement. ${ }^{53}$ However, ICD patients who had experienced a shock reported more limitation in their leisure time activities than patients in the other two groups (ICD without shock and PM) and were particularly concerned about the battery running out or about possible technical failures of their device. A greater demand for a support team in the shocked ICD group than in the nonshocked ICD group or the PM group was also reported. Thus, the authors recommend evaluating the potential benefits of a support team, for patients' families and patients themselves.

In a cross-sectional study, Newall et al compared the experience of $49 \mathrm{ICD}$ and $46 \mathrm{PM}$ patients. ${ }^{54} \mathrm{ICD}$ patients were significantly younger and were more frequently worried about their device than PM patients. Significant differences were noted between groups in the need for psychological support. The researchers also compared anxiety and depression and mental component QOL scores related to device therapy, including the number of received shocks and whether these were appropriate or not, but found no correlation between any of these variables. The authors suggested that their service helped to ensure low levels of anxiety/depression for the ICD patients, as it consisted of a small staff group whom the patients could get to know, which ensured pre-implant assessment with education both at implantation and at follow-up.

In the only identified American project involving comparison between PM and ICD patient groups, ${ }^{55}$ the authors conducted a cross-sectional correlational study using self-report data from 174 subjects (40\% pacemakers, $60 \%$ ICDs). Although no differences were noted between ICD and PM patients in total adjustment or other questionnaire scores, ICD patients were more fearful/anxious than those with a PM. This investigation showed how older people may be more accepting of physical limitations, which they regarded as natural consequences of aging, whereas younger people may feel more constrained by being unable to continue working, driving, or participating in sports that they had previously enjoyed. 
Hesse established the hypothesis that patients' misconceptions about PMs, together with inadequate psychosocial support, accounted for adjustment difficulties, and suggested a Pacemaker Support Program, to provide psychosocial counseling and education about the PM. ${ }^{56}$ Other authors proposed that the patients' perspective on their PM, and any associated lifestyle alterations, depended on the accuracy of information received on what to expect and how to be reassured that the device was normally working. ${ }^{57}$

From the patients' perspective, the most distressing issues related to the presence of an ICD are lack of detailed information, experience of multiple shocks, and an increasingly negative sensation after receiving multiple shocks. Technological improvements are expected to enhance the perceived QOL. According to this assumption, the PainFREE Rx II Trial, ${ }^{22}$ which documented the efficacy of anti-tachycardia pacing for fast VTs, demonstrated that QOL can be improved by programming anti-tachycardia pacing even in patients with fast ventricular tachycardias. With regard to a potential deterioration of perceived QOL caused by delivery of ICD shocks for fast VTs, it would be reasonable to program ATP attempts in all ICD patients, regardless of the cycle length of the index arrhythmia. Furthermore, in order to reduce the ICD interventions, an ongoing trial ${ }^{58}$ will assess the impact of higher rate cutoffs and longer delays, compared with standard programming on the burden of inappropriate therapies in primary prevention ICD and CRT-D patients. In addition, a recent study has demonstrated that patients with implanted devices continuously followed by means of the home monitoring remote control system showed a high level of acceptance of, and satisfaction with, this new technology. ${ }^{59}$

Nevertheless, ICDs can disrupt patients' psychological status and QOL, in turn promoting the pathological processes that increase the risk of sudden cardiac death; the full benefit from these devices can be achieved only when each patient's psychosocial status and QOL are preserved not only by technical/technological device-related improvements, but also by psychological support ${ }^{60}$ (Figure 6). Studies supporting interventions specifically designed to assist recovery of ICD patients have been published in recent years. While one of them found no obvious benefit from a support group intervention, ${ }^{61}$ others report the advantage of comprehensive cardiac rehabilitation programs, ${ }^{62,63}$ of a structured nursing telephone intervention, ${ }^{64}$ of a cognitive behavioral therapy-based intervention, ${ }^{65}$ of a nurse-led education program, ${ }^{66,67}$ and of a structured psychosocial management program. ${ }^{68}$

The introduction of CRT, either with or without an associated ICD, in patients with heart failure has created an additional subgroup of individuals living with quite complex pathophysiology and equally intricate technology ${ }^{69}$ Although QOL is taken in account in such patients, ${ }^{70}$ it is equally essential to inform them about the development of appropriate interventions, particularly in those considered to be 'non-responders' to the therapy. Implications of other future developments, such as remote device monitoring, ${ }^{71}$ and the increasing number of children and young adults implanted with devices, must also be considered, ${ }^{72,73}$ as it is necessary to take care of the needs of device implanted patients and of their families when facing the issue of death and dying. ${ }^{72,73}$ These

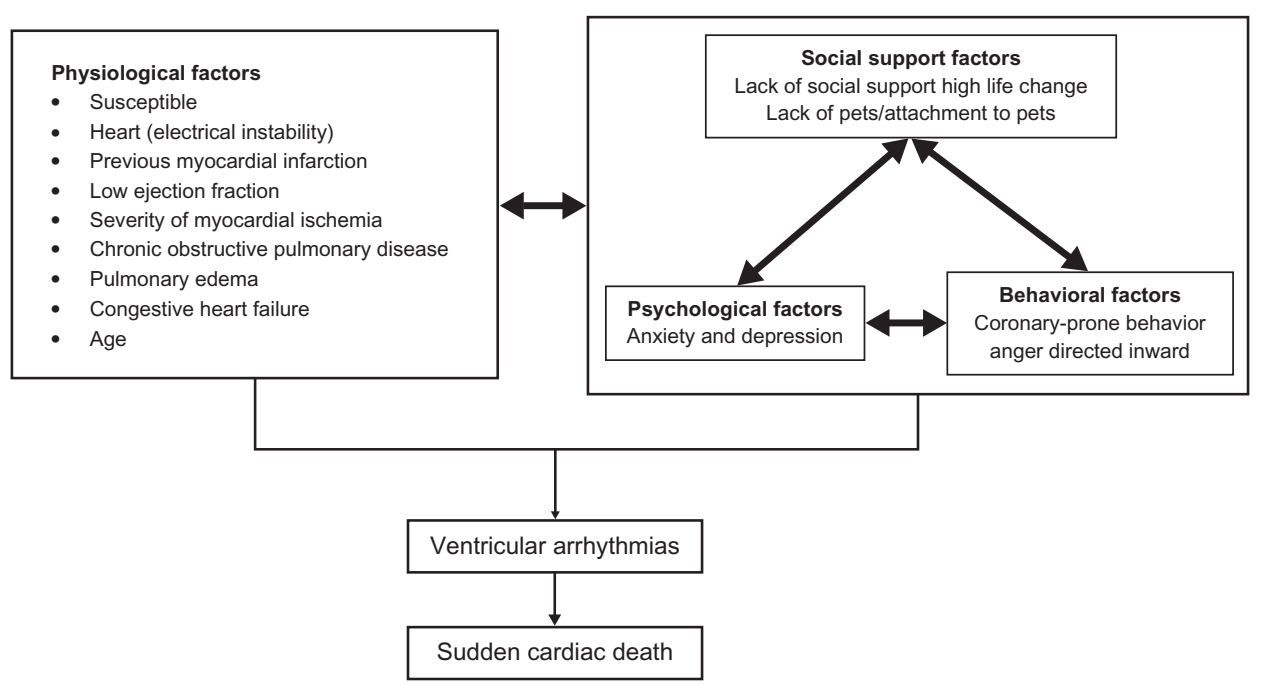

Figure 6 Holistic model of cardiovascular health. See text for explanation. 
concerns may include discussion around topics such as deactivation of shock therapies, informing palliative care teams about the symptom-control rather than the life-prolonging effects of CRT-P, and information about the need to explant the device after death prior to cremation. ${ }^{74}$ Deactivation of ICDs in patient nearing end of life is a controversial and debated issue, which is becoming increasingly topical. In this regard, the Heart Rhythm Society, in its 2008 consensus statement on CIEDs, ${ }^{75}$ affirmed that "The primary aim behind the rationale for deactivation must always be to respect the patient's right to live, or at least to die with dignity, while limiting any therapeutic action that increases the patient's level of stress, pain or anxiety." More recently, the European Heart Rhythm Society issued a consensus statement on the subject of ICDs deactivation in patients with irreversible or terminal illness. ${ }^{76}$ In this document, the members of the EHRA committee have discussed the ethical, legal and technical aspects of this issue, following the "key principles of liberal democratic societies, which include respect for the diversity of values and cultures, equal rights for all individuals, and preservation of fundamental human rights."76

\section{Conclusion}

In recent years, the rates of new implantations of ICDs and PMs have increased, due to the extended number of indications and to the population increasing aging. However, the growing burden of comorbidities in CIEDs patients, the greater complexity of the devices and the increased duration of procedures, lead to an augmented risk of infections, which is out of proportion to the increase in implantation rates. CIEDs infections are an ominous condition, which often implies the necessity of hospitalization and which carries an augmented risk of in-hospital death. Their clinical presentation may be either at pocket or at endocardial level, while in some occasions they manifest themselves only with bacteremia. For this reason, a high degree of clinical suspicion is needed, especially after detection of $\mathrm{SAB} .{ }^{26}$ Their treatment requires removal of all the implanted material, followed by an entire cycle of microorganism-specific antibiotic therapy, unless they are superficial, limited to pocket site and with no evidence of device involvement. ${ }^{25}$

Failure in CIEDs functioning is an event that, although rare, can lead to severe clinical consequences. Public authorities request the physicians to report any possible technical failure to the companies and to the authorities themselves, so that prompt and thorough information can be given to the whole medical community. Although authorities can suggest the replacement of all of the devices or leads subject to recall, in clinical practice only a minority of replacements are actually performed, while most cases are managed conservatively with close follow-up. This behavior has proved to be safe, ${ }^{48}$ as the actual number of failures is usually lower than estimated; furthermore, it is necessary to perform an accurate balance of risk with both strategies, as in some cases the removal of a potentially defective device can be more harmful than keeping it working. ${ }^{49}$

In clinical practice, patients with PMs may be perceived as requiring less support than those with ICDs, likely because their underlying cardiac condition may seem less 'acute' or 'dramatic' compared with the potential risk of cardiac arrest that indicates the need for an ICD. However, patients with ICDs and pacemakers seem to have very similar experiences. In fact, some patients with PMs are completely dependent on their device to maintain a functional heartbeat and could, therefore, be considered to have equally important support need. The main difference in how they adapt appears to be related to the experience of receiving shocks by the ICD. Both with PMs and with ICDs, it is important to assess the patients' understanding of their device and to address any gaps in knowledge, to talk about any concerns or fears related to device function or their underlying disease processes, lifestyle modifications and the implications of their current health situation in relation to their device; particular attention must be paid to ICD patients who are nearing their end of life and who might request the withdrawal of therapy. Furthermore, the emerging evidences suggest the need of specific psychological support programs, in order to interrupt or to limit the vicious circle described by the holistic model of cardiovascular health.

\section{Disclosure}

Professor Luigi Padeletti receives significant consultant fees/ honoraria from Boston Scientific Corporation, Medtronic, Saint Jude Medical and Sorin Group. The other authors have no disclosures to declare.

\section{References}

1. Zoll PM. Resuscitation of the heart in ventricular standstill by external electrical stimulation. $N$ Engl J Med. 1952;247:768-771.

2. Elmqvist R, Senning A. An implantable pacemaker for the heart In: Smyth CN, Proceedings of the Second International Conference on Medical Electronics, Paris 24-27 June 1959. London, Iliffe and Sons; 1960:253-254.

3. Mirowski M, Reid PR, Mower MM, et al. Terminations of malignant ventricular arrhythmias with an implanted automatic defibrillator in human beings. $N$ Engl J Med. 1980;303:322-324.

4. Moss AJ, Zareba W, Jackson Hall W, et al. Prophylactic implantation of a defibrillator in patients with myocardial infarction and reduced ejection fraction. $N$ Engl J Med. 2002;346:877-883. 
5. Bardy GH, Lee KL, Mark DB, et al; Sudden Cardiac Death in Heart Failure Trial (SCD-HeFT) Investigators. Amiodarone or an implantable cardioverter-defibrillator for congestive heart failure. $N$ Engl J Med. 2005;352:225-237.

6. Bristow MR, Saxon LA, Boehmer J et al. Cardiac resynchronization therapy with or without an Implantable defibrillator in advanced chronic heart failure. N Engl J Med. 2004;350:2140-2150.

7. Cleland JGF, Daubert JC, Erdmann E, et al. The effect of cardiac resynchronization on morbidity and mortality in heart failure. $N \mathrm{Engl}$ J Med. 2005;352:1539-1549.

8. Available at: http://85.255.193.60/press/ /media/F7C3D59B04AA40F6A8B6142A6FE95C46.ashx. Accessed 2011 March 6.

9. Zhan C, Baine WB, Sedrakyan A, Steiner C. Cardiac device implantation in the United States from 1997 through 2004: a population-based analysis. J Gen Intern Med. 2007;23(suppl 1):13-19.

10. Voigt A, Shalaby A, Saba S. Continued rise in rates of cardiovascular implantable electronic device infections in the United States: temporal trends and causative insights. Pacing Clin Electrophysiol. 2010;33:414-419.

11. Haddad SAP, Houben RPM, Serdijin WA. The evolution of pacemakers. IEEE Engineering in Medicine and Biology Magazine. 2006 May/ June:38-48.

12. McElderry HT, Evans JC. Pacemaker systems. In: Al-Ahmad AA, Ellenbogen KA, Natale A, Wang PJ, editors. Pacemakers and Implantable Cardioverter Defibrillators. An Expert's Manual. Minneapolis: cardiotext Publishing; 2010:3-18.

13. Lieberman R, Padeletti L, Schreuder J, et al. Ventricular pacing lead location alters systemic hemodynamics and left ventricular function in patients with and without reduced ejection fraction. $\mathrm{JAm}$ Coll Cardiol. 2006;48:1634-1641.

14. Sweeney MO, Hellkamp AS, Ellenbogen KA, et al. Adverse effect of ventricular pacing on heart failure and atrial fibrillation among patients with normal baseline QRS duration in a clinical trial of pacemaker therapy for sinus node dysfunction. Circulation. 2003;23: 2932-2937.

15. Sharma AD, Rizo-Patron C, Hallstrom AP, et al; DAVID Investigators. Percent right ventricular pacing predicts outcomes in the DAVID trial. Heart Rhythm. 2005;2:830-834.

16. Sweeney MO, Hellkamp AS. Heart failure during cardiac pacing. Circulation. 2006;113:2082-2088.

17. Sweeney MO. Algorithms for minimizing right ventricular pacing. In: Al-Ahmad AA, Ellenbogen KA, Natale A, Wang PJ, editors. Pacemakers and Implantable Cardioverter Defibrillators. An Expert's Manual. Minneapolis: Cardiotext Publishing; 2010:79-115.

18. Cannom DS, Prystowsky EN. The evolution of the implantable cardioverter defibrillator. Pacing Clin Electrophysiol. 2004; 27 : $419-431$.

19. Connolly SJ, Gent M, Roberts RS, et al. Canadian Implantable Defibrillator Study (CIDS): a randomized trial of the implantable cardioverter defibrillator against amiodarone. Circulation. 2000;101:1297-1302.

20. The Antiarrhythmics versus Implantable Defibrillators (AVID) Investigators. A comparison of antiarrhythmic - drug therapy with implantable defibrillators in patients resuscitated from near-fatal ventricular arrhythmias. $N$ Engl J Med. 1997;337:1576-1583.

21. Schaumann A, Von zur Mühlen F, Herse B, Gonska BD, Krezer H. Empirical versus tested antitachycardia pacing in implantable cardioverter defibrillators: a prospective study including 200 patients. Circulation. 98;97:66-74.

22. Wathen MS, DeGroot PJ, Sweeney MO, et al. Prospective randomized multicenter trial of empirical antitachycardia pacing versus shocks for spontaneous rapid ventricular tachycardia in patients with implantable cardioverter-defibrillators: pacing fast ventricular tachycardia reduces shock therapies (PainFREE Rx II) trial results. Circulation. 2004;110:2591-2596.

23. Sohail MR, Uslan DZ, Khan AK, et al. Risk factor analysis of permanent pacemaker infections. Clin Infact Dis. 2007;45:166-173.
24. Li JS, Sexton DJ, MicK N, et al. Proposed modification to the Duke criteria for the diagnosis of infective endocarditis. Clin Infect Dis. 2000;30:633-638.

25. Baddour LM, Epstein AE, Erickson CC, et al; American Heart Association Rheumatic Fever, Endocarditis, and Kawasaki Disease Committee; Council on Cardiovascular Disease in Young; Council on Cardiovascular Surgery and Anesthesia; Council on Cardiovascular Nursing; Council on Clinical Cardiology; Interdisciplinary Council on Quality of Care; American Heart Association. Update on cardiovascular implantable electronic device infections and their management: a scientific statement from the American Heart Association. Circulation. 2010;121:458-477.

26. Uslan DZ, Dowsley TF, Sohail MR, et al. Cardiovascular implantable electronic device infection in patients with Staphylococcus aureus bacteremia. Pacing Clin Electrophysiol. 2010;33:407-413.

27. Voigt A, Shalaby A, Saba S. Rising rates of cardiac rhythm management device infections in the United States: 1996 through 2003. J Am Coll Cardiol. 2006;48(3):590-591.

28. Uslan DZ, Sohail MR, St Sauver JL, et al. Permanent pacemaker and implantable cardioverter-defibrillator infection: a population-based study. Arch Intern Med. 2007;167:669-675.

29. Tarakji K, Chan EJ, Cantillon AJ, et al. Cardiac implantable device infections: presentation, management, and patient outcomes. Heart Rhythm. 2010;7:1043-1047.

30. Lekkerkerker JC, van Nieuwkoop C, Trines SA, et al. Risk factors and time delay associated with cardiac device infections: Leiden device registry. Heart. 2009;95:715-720.

31. Romeyer-Bouchard C, Da Costa A, Dauphinot V, et al. Prevalence and risk factors related to infections of cardiac resynchronization therapy devices. Europ Heart J. 2010;31:203-210.

32. Klug D, Balde M, Pavin M, et al; PEOPLE Study Group. Risk factors related to infections of implanted pacemakers and cardioverter-defibrillators. Result of a large prospective study. Circulation. 2007;116:1349-1355.

33. De Oliveira JC, Martinelli M, D'Orio Nishioka SA, et al. Efficacy of antibiotic prophylaxis before the implantation of pacemakers and cardioverter-defibrillators: result of a large, prospective, randomized, double-blinded, placebo-controlled trial. Circ Arrhythm Electrophysiol. 2009;2:29-34

34. Nery PB, Fernandes R, Nair GM, et al. Device-Related infection among patients with pacemakers and implantable defibrillators: incidence, risk factors, and consequences. J Cardiovasc Electrophysiol. 2010;21:786-790.

35. Al-Khatib SM, Lee Lucas F, Jollins JG, Malenka DJ, Wennberg DE. The relation between patients' outcomes and the volume of cardioverterdefibrillator implantation procedures performed by physicians treating medicare beneficiaries. J Am Coll Cardiol. 2005;46:1536-1540.

36. Viola GM, Awan LL, Darouiche RO. Non staphylococcal infections of cardiac implantable electronic devices. Circulation. 2010;121:2085-2091.

37. Knigina L, Kühn C, Kutschka I, et al. Treatment of patients with recurrent or persistent infection of cardiac implantable devices. Europace. 2010;12:1275-1281.

38. Da Costa A, Lelièvre H, Phad H, et al. Role of preaxillary flora in pacemaker infections: a prospective study. Circulation. 1998; 97:1791-1795.

39. Bloom HL, Constantin L, Dan D, et al. Implantation success and infection in cardiovascular implantable electronic device procedures utilizing an antibacterial envelope. Pacing Clin Electrophysiol. 2011;34:133-142.

40. Goldenberg I, Gillespie J, Moss AJ, et al. Long-term benefit of primary prevention with an implantable cardioverter-defibrillator: an extended 8-year follow-up study of the multicenter automatic defibrillator implantation Trial II. Circulation. 2010;122:1265-1271.

41. Gould PA, Krahn AD; Canadian Heart Rhythm Society Working Group on Device Advisories. Complications associated with implantable cardioverter-defibrillator replacement in response to device advisories. JAMA. 2006;295:1907-1911. 
42. Maisel WH. Pacemaker and ICD generator reliability: meta-analysis of device registries. JAMA. 2006;295:1929-1934.

43. Medical Device Recalls. htpp://www.fda.gov/cdrh/recalls/learn.html. Accessed 2011 June 24.

44. Carlson MD, Wilkoff BL, Maisel WH, et al. Recommendations from the Heart Rhythm society task force on device performance policies and guidelines endorsed by the ACCF, the AHA and the International Coalition of Pacing and Electrophysiology Organizations (COPE). Heart Rhythm. 2006;3:1250-1273.

45. Maisel WH. Physician management of pacemaker and implantable cardioverter defibrillator advisories. Pacing Clin Electrophysiol. 2004;27(4):437-442.

46. Kapa S, Hyberger L, Rea RF, Hayes DL. Complication risk with pulse generator change: implications when reacting to a device advisory or recall. Pacing Clin Electrophysiol. 2007;30:730-733.

47. FDA news release. FDA Preliminary Public Health Notification: Guidant VENTAK PRIZM 2 DR and CONTAK RENEWAL Implantable Cardioverter Defibrillators. http://www.fda.gov/MedicalDevices/ Safety/AlertsandNotices/PublicHealthNotifications/ucm062113.htm. Accessed 2011, June 24.

48. Perrotta L, Pieragnoli P, Ricciardi G, et al. Multicenter experience with implantable defibrillators subject to recall. Pacing Clin Electrophysiol 2011 Mar 25. [Epub ahead of print]

49. Kay GN, Brinker JA, Kawanishi DT, et al. Risks of spontaneous injury and extraction of an active fixation pacemaker lead: report of the accufix multicenter clinical study and worldwide registry. Circulation. 1999;100:2344-2352.

50. Hauser RG, Maisel WH, Friedman PA, et al. Longevity of Sprint Fidelis implantable cardioverter-defibrillator leads and risk factors for failure: implications for patient management. Circulation. 2011;123:358-363.

51. Amin MS, Matchar DB, Wood MA, Ellenbogen KA. Management of recalled pacemakers and implantable cardioverter-defibrillators: a decision analysis model. JAMA. 2006;296:412-420.

52. Priori SG, Auricchio A, Nisam S, Yong P. To replace or not to replace: a systematic approach to respond to device advisories. J Cardiovasc Electrophysiol. 2009;20:164-170.

53. Duru F, Buchi S, Klaghofer R, et al. How different from pacemaker patients are recipients of implantable cardioverter-defibrillators with respect to psychosocial adaptation, affective disorders, and quality of life? Heart. 2001;85:375-379.

54. Newall EG, Lever NA, Prasad S, Hornabrook C, Larsen PD. Psychological implications of ICD implantation in a New Zealand population. Europace. 2007;9:20-24.

55. Beery TA, Baas LS, Henthorn C. Self-reported adjustment to implanted cardiac devices. J Cardiovasc Nurs. 2007;22:516-524.

56. Hesse KA. Meeting the psychosocial needs of pacemaker patients. Int J Psychiatry Med. 1975;6:359-372.

57. Rossel CL, Alyn IB. Living with a permanent cardiac pacemaker. Heart Lung. 1977;6:273-279.

58. Multicenter Automatic Defibrillator Implantation Trial-Reduce Inappropriate Therapy, MADIT-RIT, ClinicalTrials.gov Identifier: NCT00947310.

59. Ricci RP, Morichelli L, Quarta L, et al. Long-term patient acceptance of and satisfaction with implanted device remote monitoring. Europace.12:674-679.

60. Thomas SA, Friedmann E, Kao CW, et al. Quality of life and psychological status of patients with implantable cardioverter defibrillators. Am J Crit Care. 2006;15:389-398.
61. Sneed NV, Finch NJ, MichelY. The effect of psychosocial nursing intervention on the mood state of patients with implantable cardioverter defibrillators and their caregivers. Prog Cardiovasc Nurs. 1997;12:4-14.

62. Davis LL, Vitale KA, Irmiere CA, et al. Body image changes associated with dual-chamber pacemaker insertion in women. Heart Lung. 2004;33:273-280

63. Fitchet A, Doherty PJ, Bundy C, Bell W, Fitzpatrick AP, Garratt CJ. Comprehensive cardiac rehabilitation program for implantable cardioverter-defibrillator patients: a randomised controlled trial. Heart. 2003;89:155-160.

64. Dougherty CM, Thompson EA, Lewis FM. Long-term outcomes of a telephone intervention after an ICD. Pacing Clin Electrophysiol. 2005;28:1157-1167.

65. Kohn CS, Petrucci RJ, Baessler C, Soto DM, Movsowitz C. The effect of psychological intervention on patients' long-term adjustment to the icd: a prospective study. Pacing Clin Electrophysiol. 2000;23:450-456.

66. Carlsson E, Olsson SB, Hertervig E. The role of the nurse in enhancing quality of life in patients with an implantable cardioverter-defibrillator: the Swedish experience. Prog Cardiovasc Nurs. 2002;17:18-25.

67. Smeulders ES, van Haastregt JC, Dijkman-Domanska BK, van Hoef EF, van Eijk JT, Kempen GI. Nurse- and peer-led self-management program for patients with an implantable cardioverter defibrillator: a feasibility study. BMC Nurs. 2007;6:6.

68. Sears SF, Sowell LD, Kuhl EA, et al. The icd shock and stress management program: a randomized trial of psychosocial treatment to optimize quality of life in ICD patients. Pacing Clin Electrophysiol. 2007;30:858-864.

69. Schiffer AA, Denollet J, Pedersen SS, Broers H, Widdershoven JW. Health status in patients treated with cardiac resynchronization therapy: modulating effects of personality. Pacing Clin Electrophysiol. 2008;31:28-37.

70. Mark DB, Anstrom KJ, Sun JL, et al. Quality of life with defibrillator therapy or amiodarone in heart failure. $N$ Engl $J$ Med. 2008;359:999-1008.

71. Lunati M, Gasparini M, Santini M, et al. Follow-up of CRT-ICD: implications for the use of remote follow-up systems. Data from the insyncicditalian registry. Pacing Clin Electrophysiol. 2008;31:38-46.

72. Alexander ME, Cecchin F, Walsh EP, Triedman JK, Bevilacqua LM, Berul CI. Implications of implantable cardioverter defibrillator therapy in congenital heart disease and pediatrics. $J$ Cardiovasc Electrophysiol. 2004;15:72-76.

73. Sears SF, Conti JB. Implantable cardioverter-defibrillators for children and young adolescents: mortality benefit confirmed-what's next? Heart. 2004;90:241-242.

74. Goldstein NE, Lampert R, Bradley E, Lynn J, Krumholz HM. Management of implantable cardioverter defibrillators in end-of-life care. Ann Intern Med. 2004;141:835-838.

75. Wilkoff BL, Auricchio A, Brugada J, et al. HRS/EHRA expert consensus on the monitoring of cardiovascular implantable electronic devices (CIEDs): description of techniques, indications, personnel, frequency and ethical considerations. Europace. 2088;10:707-725.

76. Padeletti L, Arnar DO, Boncinelli L, et al. EHRA Expert Consensus Statement on the management of cardiovascular implantable devices in patients nearing end of life or requesting withdrawal of therapy. Europace. 2010;12:1480-1489.
Medical Devices: Evidence and Research

\section{Publish your work in this journal}

Medical Devices: Evidence and Research is an international, peerreviewed, open access journal that focuses on the evidence, technology, research, and expert opinion supporting the use and application of medical devices in the diagnosis, treatment and management of clinical conditions and physiological processes. The identification of novel

\section{Dovepress}

devices and optimal use of existing devices which will lead to improved clinical outcomes and more effective patient management and safety is a key feature. The manuscript management system is completely online and includes a quick and fair peer-review system. Visit http://www. dovepress.com/testimonials.php to read real quotes from authors. 